\title{
Caracterizaciones de género en la divulgación de la cardiopatía isquémica
}

\author{
María José RuIz SomaVILla \\ Universidad de Málaga (España) \\ rsomavilla@uma.es \\ Gabriel NARBONA CARRIóN \\ Centro de Salud Victoria (España) \\ narmo.6@hotmail.com \\ Carmen AgüEra Urbano \\ Hospital Costa del Sol (España) \\ caguera@gmail.com
}

\begin{abstract}
Resumen:
Este trabajo aborda discursos y códigos de género sobre la cardiopatía isquémica proyectados por la prensa en las dos últimas décadas del siglo XX. En el examen del discurso divulgativo se han identificado estereotipos de género en la explicación de la morbilidad y la mortalidad en hombres y mujeres, y estrategias sustentadas en el discurso de la domesticidad que vincularon la enfermedad con la transgresión y actuaron como mecanismos de subalternidad.
\end{abstract}

Palabras clave: Divulgación, cardiopatía isquémica, género, prensa, sesgos, estereotipos

\section{Gender characterizations in the popularization of ischemic cardiomyopathy}

\begin{abstract}
:
This article deals with discourses and gender codes related to ischemic cardiopathy spread by the press in the last two decades of the $20^{\text {th }}$ century. In the analysis of the popularizing discourse that explains the morbidity and the mortality in men and women, gender stereotypes have been identified, as well as strategies that were based on the discourse of domesticity that connected illness with transgression and worked as mechanisms of subalternity.
\end{abstract}

Key Words: Popularization, ischemic cardiomyopathy, gender, press, bias, stereotypes

Referencia normalizada:

Ruiz Somavilla, M. J.; Narbona Carrión, G.; Agüera Urbano, C. (2013) Caracterizaciones de género en la divulgación de la cardiopatía isquémica. Historia y Comunicación Social. Vol. $18 \mathrm{~N}^{\mathrm{o}}$ Especial Octubre. Págs. 591--602

Sumario: 1. Introducción. 2. El azote del hombre moderno. 3. De las hormonas protectoras a la emancipación. 4. A modo de conclusión. 


\section{Introducción}

En el último tercio del siglo XX se vinculó la patología coronaria al desarrollo socioeconómico y se consolidó la noción de enfermedad de la riqueza que afectaba a los hombres, especialmente a los ejecutivos. A ello contribuyó el sesgo en la investigación que introdujo la Food and Drug Administration, al proponer en 1977 la exclusión de las mujeres en edad fértil de los ensayos clínicos, y los sesgos de género en el diagnóstico y el tratamiento de las mujeres con cardiopatía isquémica (Lerner, 1986; Bird, 1999; Miqueo, 2004; Ruiz Cantero, 2004).

En los primeros años de la década de los noventa se denunció que el número de mujeres diagnosticadas de esta enfermedad podía ser diferente a los casos reales. Era necesario determinar si los procedimientos diagnósticos y terapéuticos que se ponían en marcha ante un infarto de miocardio eran los mismos en hombres y mujeres (Healy, 1991). Desde este presupuesto se indagó en las historias clínicas con el objeto de determinar la frecuencia real de la enfermedad en las mujeres, y se observó que la frecuencia de algunas consecuencias clínicas de la patología isquémica igualaba o incluso superaba a la de los hombres (Ayanian, 1991; Marrugat, 1994). Al confirmarse una discrecionalidad en la aplicación de los procedimientos y su desigual eficacia, se denunció la presencia de sesgos de sexo y de género en la investigación de la patología cardíaca, y se cuestionó el carácter masculino de la enfermedad y su vinculación con una posición socioeconómica alta (Castelli, 1988; Agencia de Evaluación de Tecnologías Sanitarias, 2001; Schoenberg, 2003; Rohlfs, 2004; Ruiz-Cantero, 2004; Ridker, 2005; Aguado-Romero, 2006). Se podría suponer que estos nuevos conocimientos, trasmitidos desde la medicina y los estudios de género, desplazaron las creencias sobre la presentación casi exclusiva de la enfermedad en los hombres de las sociedades más desarrolladas. Sin embargo, lejos de cuestionarse, esas creencias se han ido reafirmando y han permanecido en el imaginario colectivo hasta llegar a la actualidad ${ }^{1}$.

En el proceso de construcción de las representaciones sobre la cardiopatía isquémica han intervenido los medios de comunicación divulgando el conocimiento generado por la medicina. Este trabajo examina los discursos y códigos de género de esos mensajes de divulgación, centrándonos en las estrategias utilizadas en el proceso de recontextualización del discurso médico. Para ello se ha realizado una revisión sistemática de entrevistas a cardiólogos, reportajes, noticias de jornadas, seminarios, congresos; artículos de opinión y noticias, en prensa escrita (ABC, Hoja de Lunes, Mediterráneo) y revistas (Flores y abejas y Blanco y Negro), durante las dos últimas décadas del siglo XX.

\section{El azote del hombre moderno}

La medicina había construído la cardiopatía isquémica, especialmente el infarto de miocardio, como una enfermedad de las sociedades postindustriales ligada al desa- 
rrollo y al bienestar. Los datos parecían indicar que la cardiopatía isquémica era la primera causa de muerte en estos países. A lo largo del período estudiado es constante la aparición de noticias de muertes por infarto de miocardio de personajes populares, o con alguna relevancia social, a la vez que los estudios médicos ponían en evidencia el aumento de la morbilidad y mortalidad a causa de esta enfermedad. Todo ello fue creando tal clima de alarma que en las páginas de los medios analizados se llegaba a calificar a la cardiopatía isquémica como la epidemia, la plaga o el azote de los hombres de finales del siglo XX (ABC Sevilla, 14-10-80, ABC, 22-10-86; 6-11-88; 24-2-88). La preocupación sanitaria llevó a considerar el carácter social y económico del problema para procurar respuestas más eficaces $(A B C, 9-5-86$; ABC Sevilla, 18-12-86). Había que determinar los factores de riesgo para evitar que creciese el número de afectados. Estos estudios médicos se trasladaron al campo de la divulgación y, aunque se planteó como un problema social y cultural, se dirigió la atención a las medidas preventivas de carácter individual. La presencia de la enfermedad indicaba una falta de higiene previa en la atención a la salud, por desconocimiento de los factores de riesgos ( $A B C, 1-3-89)$.

El abordaje de los factores de riesgo de la cardiopatía isquémica por la medicina fue ampliamente difundido por los medios de comunicación. Desde principio de los ochenta se informó de las campañas de divulgación en televisión, radio y prensa, y en los periódicos y revistas analizados se exponían los resultados de esos trabajos sobre los riesgos del tabaco, el sedentarismo, la dieta, la genética, los niveles de colesterol y de presión arterial en el desarrollo de la enfermedad. En la elaboración de estas noticias se puede advertir una visión cientificista de la actividad médica, en la que, entre otros, está presente el mito del experto ${ }^{2}$. $\mathrm{Al}$ asumir que la opinión sobre las cuestiones pertenecientes a un determinado campo científico sólo le corresponde a los expertos de ese campo, las noticias se presentaban desde la autoridad de los cardiólogos. En general, el comunicador se limitaba a presentarlos e introducir sus trabajos o sus explicaciones. Por eso, la mayor parte de la información sobre los factores de riesgo procedía de entrevistas a estos profesionales de la medicina, y sus palabras, sin reconstrucción ni mediación, constituyeron el mensaje transmitido a los lectores. Con esta utilización de la figura del experto la información quedaba legitimada por su prestigio (Salvador, 2002).

En este discurso se expresaba que la causa del infarto de miocardio había que buscarla en la actividad laboral desarrollada por los ejecutivos, hombres de negocios de elevado estatus socioeconómico. Con ello, los protagonistas del desarrollo se convertían en las víctimas de una actividad vinculada al éxito social y económico. Esta actividad se había relacionado con la cardiopatía isquémica mediante la conceptualización de la personalidad tipo $\mathrm{A}^{3}$. Se trataba de un estereotipo masculino caracterizado por una conducta agresiva, ambiciosa, competitiva, incapaz de perder el tiempo ("caminan rápido, comen deprisa y realizan actividades distintas simultáneamente"), con tendencia a la hostilidad y a la ira. El ejecutivo "quiere llegar a un tope y abarcarlo todo lleno de ansiedad" y de estrés. Este último concepto se entendía como el sobreesfuerzo constante por llevar un ritmo vertiginoso de vida, abrumado y 
sobrepasado, permanentemente desbordado y sin tiempo para casi nada ( $A B C, 13-12-$ $86,1-10-89 ; 18-11-90$ y 3-3-92). La ilustración que acompaña a la información sobre la frecuencia, la fisiopatología y la terapéutica trombolítica del infarto de miocardio muestra nítidamente quién era la diana de esta patología. El dibujo muestra un hombre de edad media, vestido con traje y corbata, que se lleva la mano a la región precordial, y parece doblarse de dolor a la vez que se le cae el maletín. De fondo, el trazado de un electrocardiograma no deja lugar a dudas: ese hombre está sufriendo un infarto de miocardio. El reportaje con la ilustración se publicó el 5 de octubre de 1988 en $A B C$ de Madrid y cuatro días después en el de Sevilla. Un año después, la ilustración vuelve a aparecer para reforzar la idea del temor masculino a padecer la enfermedad, debido "al miedo por la dejación de la familia que no se da cuenta del mal que produce el exceso de trabajo; el desasosiego que produce en el enfermo la posible pérdida de lo conseguido con tanta dedicación y, por último, la decepción que causa el no poder disfrutar el éxito que tanto ha costado" $(A B C, 1-10-89)$. De nuevo vuelve a aparecer la ilustración en 1995 ( $A B C, 19-10-95)$. La prensa se hacía eco de las medidas propuestas por la medicina para prevenir la enfermedad, anunciando mesas redondas y conferencias, como la convocada en 1985 sobre "La prevención del infarto de miocardio en el directivo de empresa", o la información aparecida en 1988 sobre una conferencia impartida en la Asociación Española de Científicos titulada "Las enfermedades de los ejecutivos", en la que se habló de los factores de riesgo que conducían inevitablemente al infarto de miocardio ( $A B C, 29-11-85$ y 23-7-88).

Sin embargo, se había abierto una línea de investigación médica que estaba mostrando la carencia de validez de este modelo. Se advertía que en los países desarrollados los grupos socieconómicos menos favorecidos tenían un mayor riesgo de padecer cardiopatía isquémica ${ }^{4}$. Algunas sociedades médicas adoptaron medidas en este sentido, como las intervenciones programadas por la Fundación Andaluza de Ayuda a la Cardiología, en colaboración con los medios de comunicación, dirigidas a todos los estamentos sociales, o el reparto de folletos y trítpticos, en los ambulatorios y otros centros sanitarios $(A B C, 20-10-1987)$. En 1992 apareció una noticia sobre el XXVII Congreso de la Sociedad Andaluza de Cardiología, en cuyo acto de presentación los cardiólogos señalaron a la pobreza, la marginación, el paro y el bajo nivel educativo como factores relacionados con la aparición del infarto de miocardio $(A B C, 26-11-92)$. Pero esta información no tuvo eco en los artículos de opinión ni en los reportajes, ni se volvió a incidir sobre este aspecto en las páginas de los medios analizados.

En los años que abarca nuestro estudio se mantuvo vigente la imagen de unos ejecutivos que podían enfermar por una actividad laboral relacionada con actitudes y comportamientos considerados masculinos, mientras que las mujeres permanecían ajenas a estos problemas de salud. Se estaba reforzando la idea de que la causa de la menor morbilidad y mortalidad estaba en la propia naturaleza femenina. La metáfora del contagio se utilizó para naturalizar la ausencia de enfermedad: "ellas son mucho más inmunes a las enfermedades que su varonil compañero. Lo comprobamos en la terrible enfermedad del cáncer... Y otro tanto puede decirse de las enfermedades 
cardiacas, donde el infarto de miocardio es casi privativo del hombre" (Mediterráneo, 1-11-83 y 1-11-84). Las mujeres podían despreocuparse de la enfermedad.

Por tanto, las mujeres podían vivir con la tranquilidad de que su naturaleza y su rol social las mantenían alejadas de las manifestaciones clínicas de la cardiopatía isquémica. Incluso el relato de los síntomas que debían hacer pensar en un infarto, contribuyó a mantener ese distanciamiento con la enfermedad. Cuando los médicos comprobaron que el retraso en el tratamiento limitaba las posibilidades de curación, dedicaron su atención en las páginas de los medios a dar a conocer los síntomas, transmitiendo la necesidad de estar alerta ante la aparición de dolor o sensación de opresión localizado a la altura o en el área "de la corbata" (Flores y abejas, 30-1-85; $A B C, 20-5-87$ y 1-10-89). Parece evidente que las mujeres no se sintieran concernidas con estas palabras, lo que indudablemente contribuía a subestimar el riesgo de padecer la enfermedad. Hasta tal punto estaba distorsionada la incidencia de la enfermedad en las mujeres que, para tratar de erradicar el miedo a la actividad sexual después de haber sufrido un infarto, se advertía que "la mujer de aquel que ha sufrido un infarto suele encontrarse abrumada por la responsabilidad, tiende con frecuencia a evitar el contacto sexual con su marido, y, si lo realiza se siente culpable". No creyeron necesario advertir a los maridos cómo actuar en el caso de que sus mujeres hubiesen sufrido un infarto $(A B C, 24-11-87)$. Lo que tampoco es de extrañar puesto que, para estos médicos y periodistas, la cardiopatía isquémica era una enfermedad masculina. Además, en la nota titulada "Maridos candidatos al infarto" en la sección de anuncios de Mediterráneo (5-8-83), se puede apreciar como se hacía intervenir en el problema otra variable: las propias las mujeres. Se informó del elevado riesgo de padecer infarto los hombres cuyas mujeres trabajan fuera de casa -el doble o el triple que el resto-. Las causas había que atribuirlas a unas mujeres liberadas que además de amenzar la autoestima del marido por la actividad que desempeñaban, trasladaban las tensiones de su vida laboral al hogar.

\section{De las hormonas protectoras a la emancipación}

Cuando se argumentó la ausencia de enfermedad en las mujeres se caracterizaron elementos vinculados al sexo y al género como factores de riesgo de cardiopatía isquémica. Se asoció la ausencia de enfermedad con la fisiología femenina y comportamientos considerados de género. Por ejemplo, las mujeres estarían menos expuestas a los riesgos de las enfermedades coronarias por tener mayor cantidad de alfa-lipoproteína que los hombres, pero, sobre todo, porque estaban protegidas por sus hormonas. Se vinculó la presencia de la enfermedad con los cambios hormonales de la menopausia pues, según los datos, las mujeres se diagnosticaban de cardiopatía isquémica a partir de los sesenta y cinco años: "la mujer dispone de una protección hormonal y no tiene angina de pecho ni cardiopatía coronaria hasta después que deja de tener reglas" (Blanco y Negro, 14-6-92). 
A finales del siglo XX se adelantó la edad de aparición de enfermedad coronaria en las mujeres por dos razones. Desde la perspectiva fisiopatológica se cuestionó el mito de los efectos beneficiosos y protectores de las hormonas sobre el sistema cardiovascular, que terminó por desmontarse con la información aportada en el XXI Congreso Europeo de Cardiología, celebrado en Barcelona. En las páginas de la prensa se difundió una noticia que parecía inquietante: los estrógenos no protegían el corazón tras la menopausia $(A B C, 2-9-99)$. Pero desde una perspectiva de género, la justificación del aumento de diagnósticos de patología coronaria antes de la menopausia fue demoledora para las mujeres. La prensa y las revistas analizadas dieron a conocer que un sector de la investigación médica había relacionado la presencia de la enfermedad en las mujeres con la adopción de conductas, comportamientos y roles masculinos en el desempeño de su actividad laboral. Estas ideas no eran nuevas, pues a comienzos de los ochenta, con motivo del XVII Congreso de la Sociedad Española de Cardiología, se informó que el número de afectados por las enfermedades cardiovasculares iba en aumento, y que se había producido a costa de los jóvenes y las mujeres, que ya habían empezado a someter "el organismo a las mismas agresiones que los hombres principalmente el tabaco y el alcohol" (ABC Sevilla, 4-11-81). Pero, ahora, eran los caracteres asociados a las diferencias de género los que centraban la atención de un discurso que encontró en la cardiopatía isquemica una herramienta para transmitir conceptos esenciales del discurso de la domesticidad. Sectores de la medicina y del periodismo compartían nociones y estereotipos asociados a los roles de género tradicionales, como el del "ángel del hogar" (Jagoe, 1998), un modelo que se había empezado a sustituir en las primeras décadas del siglo XX por el de la "Nueva Mujer Moderna". Sin embargo, en España, al ser adoptado por el franquismo, tuvo continuidad en determinados discursos y prácticas (Ortiz Gómez, 2001; Romo Parra, 2002; Nash, 2004).

A finales de los noventa, el aumento de la mortalidad por infarto en mujeres se atribuía al tabaco, al abandono de la dieta mediterránea y a la progresiva incorporación de las mujeres al ámbito laboral (ABC Sevilla, 20-3-96). En el proceso de recontextualización de este mensaje científico hemos podido detectar algunos problemas, como la conversión de la información en noticia-espectáculo, con la trivialización y banalización de los contenidos, y mecanismos de distorsión del mensaje científico. La trivialización de los temas tratados atraviesa el mensaje divulgativo, especialmente las conceptualizaciones de las mujeres con mayores probabilidades de enfermar. A finales de la década de los ochenta se planteó la existencia de un nuevo factor de riesgo de cardiopatía isquémica en las mujeres: la "conducta de emancipación". El infarto de miocardio aparecía entonces como el precio que pagaban las mujeres por emanciparse e incorporarse a actividades consideradas masculinas. Esta idea aparecía justificada en estudios como el del Hospital Clínico de la Universidad de Erlangen, realizado entre 237 "pacientes femeninas emancipadas" que habían sufrido infarto de miocardio: "El estudio ha evidenciado que las mujeres entre veintiuno y treinta años con un alto nivel intelectual y ambiciosas tendencias hacia la independencia y la emancipación reúnen en gran escala los factores de riesgo de infarto conocidos" $(A B C, 18-4-88)^{5}$. Se transmitía que las mujeres se mantendrían alejadas de la 
enfermedad si cumplían el papel social tradicional hasta llegar a la menopausia. Por el contrario, la transgresión de ese rol y la adopción de actitudes y comportamientos considerados masculinos, las aproximaba también a la enfermedad. Es evidente que la presencia creciente de estas mujeres en espacios tradicionalmente masculinos, alarmó a determinados sectores de la sociedad. Los periodistas y divulgadores sensibles a esta situación pusieron en marcha esta estrategia de comunicación para defender valores relacionados con el modelo de género tradicional y desautorizar actitudes y comportamientos desviados de ese modelo. Es evidente que esta información podría haberse expresado en otros términos. No parece necesario insistir en la intensidad del prejuicio sexista que supone considerar ambicioso el deseo de ser independiente o de emanciparse, como queda patente en el uso de la expresión "pacientes femeninas emancipadas".

Este discurso legitimador de un orden social que ya no tenía sustento en la estructura social, política, legal y cultural de finales del siglo XX se revitalizó unos años más tarde, con un nuevo estudio realizado en Suecia. A pesar de haberse alcanzado la equidad de género en el terreno legal, los mecanismos de subalternidad, la desigualdad y la subordinación, se mantenían en sectores sociales como el que elaboró y difundió estas representaciones culturales de la feminidad que, como señalamos, seguían vinculadas al discurso de la domesticidad. En Suecia habían observado una elevada mortalidad de origen cardiaco en la población femenina. La administración puso en marcha un estudio para averiguar si ese incremento de muertes obedecía a factores de origen colectivo, y los resultados los dio a conocer la Comisión de Ambiente Laboral en 1992. La autora del reportaje de $A B C$ reducía el problema a la actitud de estas mujeres: "la mujer sueca se mata trabajando". La causa de la elevada y precoz mortalidad por infarto de miocardio era la intensa hiperactividad de unas mujeres que, entre los cuarenta y sesenta y cinco años empezaban a ser "ser víctimas de enfermedades típicamente masculinas, como el infarto". Planteaba que se estaban cuestionando si hogar y trabajo eran compatibles, y si las mujeres debían abandonar la actividad laboral para vivir más años $(A B C, 4-5-92)$ :

"Resulta curioso que esa «perla rubia» que manda en casa y en las empresas (...) aventaja ya a los hombres incluso a la hora de morir, debido al estrés, las enfermedades cardiovasculares y el infarto de miocardio. El número de mujeres que rompe su corazón por una superactividad laboral ha aumentado en un 52 por 100 durante la última década. ¿Puede asociarse el trabajo remunerado femenino con un deterioro para la salud? Tal vez, pues esas modernas vikingas que desde hace tiempo han invadido el mercado laboral, ocupando gran parte de los puestos de ejecutivos, trabajan hasta morir por causa de una tremenda ambición de ser las mejores (...) El riesgo es mayor si esa fémina bebe y fuma «como un macho»".

La corresponsal informaba sobre lo que podría ser una preocupación médica, el incremento de la mortalidad en las mujeres a causa de su ritmo de trabajo y falta de descanso, algo que, según la misma corresponsal, no parecía preocupar a esas mujeres. Además, la caracterízación que acabamos de ver termina por cerrar el círculo de la transgresión, al decir que esa mujer manda, rompe, invade, ocupa, verbos tradicionalmente vinculados a una identidad masculina propia del simbolismo de género. 
No obstante, la culpabilización de estas mujeres no era absoluta puesto que, a las consecuencias de realizar una actividad laboral remunerada, se sumaba la falta de descanso motivada por razones biológicas. En el reportaje se explicaba las diferencias en los niveles de cortisol entre hombres y mujeres. Señalaba que, en las mujeres, se producía un aumento por la noche, en cambio en los hombres había un descenso. Afirmaba que en estas diferencias hormonales residía la causa del comportamiento masculino y femenino al llegar a casa después de la jornada laboral: los hombres cenan y ven la televisión y las mujeres hacen la cena, lavan, planchan y acuestan a los niños. Es patente el reduccionismo biológico de este discurso, que utiliza unos niveles hormonales para legitimar la separación de tareas y la adscripción de la mujer al trabajo doméstico, y la puesta en marcha de la estrategia que hace encajar los datos procedentes de los estudios médicos en el discurso ideológico sobre el papel social de las mujeres.

Por otra parte, estos datos de 1992 guardan relación con dos artículos de 1980 publicados en revistas médicas internacionales, a partir de uno de los mayores estudios sobre enfermedad cardiovascular. En ellos se indicaba que la tasa de enfermedad coronaria era casi el doble en las mujeres que desempeñaban trabajos de oficina, y se exponía la importancia de los factores psicosociales en el desarrollo de la enfermedad coronaria en las mujeres ${ }^{6}$. Sin embargo, hay una diferencia fundamental entre estos trabajos y los artículos de divulgación que estamos analizando, ya que los estudios médicos de 1980 no incorporaron los estereotipos de género y los sesgos sexistas que acabamos de advertir en el mensaje divulgativo. Estos estudios médicos sobre el carácter biopsicosocial de la cardiopatía isquémica y los que estaban mostrando su incidencia real en las mujeres, alertaron sobre la vinculación de la enfermedad con las consecuencias derivadas de las identidades de género, los roles, ocupaciones y obligaciones familiares y laborales (Rohlfs, 2007). Una sobrecarga física y emocional que se agudizaría en situaciones de pobreza y exclusión social.

Estas investigaciones que trataban de hacer visible la enfermedad en las mujeres evitando los sesgos de sexo y de género, habían ofreciedo resultados que alarmaron a un sector importante de la medicina. Aunque se conocía que la cardiopatía isquémica era una causa importante de morbilidad y mortalidad en las mujeres y se sabía de la relación entre mortalidad y demora en la solicitud de asistencia y que este retraso podía relacionarse con una presentación "atípica" de la enfermedad, no fue hasta finales de los noventa cuando aparecieron estas ideas en la prensa y las revistas analizadas (Blanco y Negro, 8-2-98; ABC, 27-7-95 y 21-1-99).

\section{A modo de conclusión}

El mensaje divulgativo sobre la cardiopatía isquémica adoptó acríticamente y difundió creencias y estereotipos del discurso de la domesticidad que manejaban médicos y periodistas. Los trabajos médicos que cuestionaban el carácter masculino 
de la cardiopatía isquémica y los que plantearon la existencia de sesgos de sexo y de género en la investigación, el diagnóstico y el tratamiento apenas encontraron cabida en medios analizados. Por ello la modificación en las cifras de morbilidad y mortalidad en las mujeres se vinculó al cambio en su rol social, ocultando que la enfermedad se había hecho invisible para la medicina a causa de esos sesgos y estereotipos. Al integrar la información médica en un modelo ideológico normativo, la distorsión, el reduccionismo, los procedimientos de mitificación y otras estrategias de comunicación actuaron como mecanismos de subalternidad en el proceso de recontextualización de los mensajes.

\section{Bibliografía}

AGUADO-ROMERO, M.J.; MÁRQUEZ-CALDERON, S.; BUZÓN-BARRERA, M.S. (2006). “¿Sesgo de género en la actuación hospitalaria frente al síndrome coronario agudo?" Revista Española de Cardiología, 59, 785-793.

AYANIAN, J.Z.; EPSTEIN, A.M. (1991). "Differences in the use of procedures between women and men hospitalized for coronary heart disease". New England Journal of Medicine, 325(4), 221-225.

BIRD, C.E.; RIEKER, P.P. (1999). "Gender matters: an integrated model for understanding men's and women's health". Social Science \& Medicine, 48, 745-755.

CALVO HERNANDO, M. (1992). Periodismo cientifico. Madrid: Paraninfo.

CASTELLI, W.P. (1988). "Cardiovascular disease in women". American Journal of Obstetrics \& Gynecology, 158(6), 1553-1560.

GOLD, M.R.; FRANKS, P. (1990) "The social origin of cardiovascular risk: an investigation in a rural community". Int J Health Serv. 20 (3), 405-16.

HEALY, B. (1991). "The Yentl syndrome". New England Journal of Medicine, 325, 274-276.

JAGOE, C.; BLANCO, A.; ENRÍQUEZ DE SALAMANCA, C. (1998). La mujer en los discursos de género. Barcelona: Icaria.

LERNER, D.; KANNEL, W. (1986). "Patterns of coronary heart disease morbidity and mortality in the sexes: A 26-year follow-up of the Framingham Study". American Heart Journal, 111(2), 383-390.

MARRUGAT, J; ANTÓ, J.M.; SALAS, J. et al. (1994). "Influence of gender in acute and long-term cardiac mortality after a first myocardial infarction". Journal of Clinical Epidemiology, 47, 111-118.

MIQUEO, C. (2004). "Genealogía de los sesgos de género en la ciencia y la práctica médica contemporánea”. En Martín Pérez, J. (coord.): La medicina ante el nuevo milenio: una perspectiva histórica. Cuenca: Universidad de Castilla La Mancha, pp. 45-66.

MORRISON, C.; WOODWARD, M.; LESLIE, W. et al. (1997) "Effect of socioeconomic group on incidence of, management of, and survival after myocardial infarction and coronary death: analysis of community coronary event register". $B M J, 314(7080), 541-546$. 
MOSER, K.A.; FOX, A.J.; GOLDBLATT, P.O. et al. (1986) "Stress and heart disease: evidence of associations between unemployment and heart disease from the OPCS Longitudinal Study". Postgrad Med J, 62(730), 797-799.

NASH, M. (2004). Mujeres en el mundo. Historia, retos y movimientos. Madrid: Alianza.

NELKIN, D. (1990). La ciencia en el escaparate. Madrid: Fundesco.

ORTIZ GÓMEZ, T. (2001). "El género, organizador de profesiones sanitarias". En Miqueo, C. et al.: Perspectivas de género y salud. Madrid: Minerva.

ROMO PARRA, C. (2002). "Crecimiento económico y universo privados". En Ramos, M.D. et al. (coords.): Discursos, realidades, utopías. La construcción del sujeto femenino en los siglos XIX y XX. Barcelona: Anthropos.

ROQUEPLO, P. (1983). El reparto del saber. Barcelona: Gedisa.

RUIZ SOMAVILLA, M.J. (en prensa). "Género y salud en la investigación biomédica y la medicina clínica. Enfermedades ocultas y mujeres transparentes". En Postigo, M. et al. (coords): Voces consonantes feministas desde las humanidades, las ciencias sociales y experimentales. Málaga: Universidad de Málaga.

SCHOENBERG, N.E.; PETERS, J.E.; DREW, E.M. (2003). "Unraveling the mysteries of timing: women's perceptions about time to treatment for cardiac symptoms". Social Science \& Medicine, 56, 271-284.

WOODWARD, M.; SHEWRY, M.C.; SMITH, W.C. et al. (1992) "Social status and coronary heart disease: results from the Scottish Heart Health Study". Prev Med. 21(1),136-148.

Publicaciones web

ALCIBAR, M. (2009). "Comunicación pública de la tecnociencia: más allá de la difusión del conocimiento", en Zer, 14(27). Disponible en: http://www.ehu.es/zer/ hemeroteca/pdfs/zer27-08-alcibar.pdf. [14-7- 2013].

AGENCIA DE EVALUACIÓN DE TECNOLOGÍAS SANITARIAS (2001). Manejo hospitalario de la cardiopatía isquémica en España. Análisis de situación. Madrid: Ministerio de Sanidad y Consumo. Disponible en: http://gesdoc.isciii. es/gesdoccontroller?action=download\&id=07/11/2012-ffbdbf0696. [17-7-2013].

BELENGUER JANÉ, M. (2003). "Información y divulgación científica: dos conceptos paralelos y complementarios en el periodismo científico", en Estudios sobre el Mensaje Periodístico, $\mathrm{n}^{\circ} 9$. Disponible en: http://dialnet.unirioja.es/servlet/articulo? codigo $=860254$ \&orden $=1$ \&info $=$ link. [16-6-2012].

CUARTAS MURILLO, A.; ESCOBAR ZULOAGA, A.; LOTERO GARCÍA, E. et al. (2008). "Los factores psicosociales implicados en la cardiopatía isquémica". Revista CES Psicología, 1(2). Disponible en: http://factorespsicosociales.com/ articulos/?p=738. [20-5-2011].

HERNANDO CUADRADO, Luis A. (2006). "Periodismo científico y lenguaje", en Estudios sobre el Mensaje Periodístico, 12, 331-348. Disponible en: http://revistas.ucm.es/index.php/ESMP/article/view/ESMP0606110331A. [12-6-2013]. 
Informe sobre la situación mundial de las enfermedades no trasmisibles 2010 (2011). OMS. Disponible en: http://www.who.int/nmh/publications/ncd_report_ summary_es.pdf. [17-7-2013].

NASH, M. (2006). "Identidades de género, mecanismos de subalternidad y procesos de emancipación femenina", en Revista CIDOB d'Afers Internacionals, $\mathrm{n}^{\circ}$ 73-74. Disponible en: http://dialnet.unirioja.es/servlet/revista?codigo=2496. [15-6-2011] RIDKER, P.M.; COOK, N.R.; LEE, I.M. et al. (2005). "A Randomized Trial of Low-Dose Aspirin in the Primary Prevention of Cardiovascular Disease in Women", en The New England Journal of Medicine, 352(13). Disponible en: http://www.nejm.org/doi/pdf/10.1056(NEJMoa050613. [2-6-2011].

ROHLFS, I.; GARCÍA, M.M.; GAVALDÀ, L., et al. (2004). "Género y cardiopatía isquémica", en Gaceta Sanitaria [online], 18(supl.2). Disponible en: http://scielo. isciii.es/pdf/gs/v18s2/revision6.pdf. [2-6-2011].

ROHLFS, I. (2007): "Género y salud: diferencias y desigualdades". Disponible en: http://www.raco.cat/index.php/quark/article/viewFile/54973/65434. [4-5-2011]

RUIZ-CANTERO, M.T.; VERDÚ-DELGADO, M. (2004). "Sesgo de género en el esfuerzo terapéutico", en Gaceta Sanitaria [online], 18(supl.1). Disponible en: http://scielo.isciii.es/pdf/gs/v18s1/03sesgo.pdf. [2-6-2011].

SALVADOR, V. (2002). "Discurso periodístico y gestión social de los conocimientos: algunas observaciones sobre la didacticidad", en Anàlisi, 28, 107-120. Disponible en: http://cei.udc.es/uploads/archivos/files/[2]\%20Didacticitat(2).pdf. [16-6-2012].

SHARMA, K.; GULATI, M. (2013). "Coronary Artery Disease in Women. A 2013 Update". Global heart, 8(2), 105-112. Disponible en: http://0-www.sciencedirect. com.jabega.uma.es/science/journal/22118160/8/2. [30-9-13].

\section{Notas}

1 Puede verse el Informe sobre la situación mundial de las enfermedades no trasmisibles, 2011. Según un estudio reciente, a pesar de que la mortalidad por enfermedad de la arteria coronaria es mayor en mujeres, la probabilidades de recibir consejos para prevenir la enfermedad, para tratar los niveles de colesterol y para que se les prescriba rehabilitación después de un infarto de miocardio, continúa siendo menor que en los hombres (Sharma, 2013).

2 Nos estamos refiriendo a los mitos cientificistas enunciados por el Grupo Survivre. Puede verse Miguel Alcíbar (2009).

3 Rosenman y Friedman describieron en 1959 ese patrón de conducta en el que predominan las estrategias agresivas para conseguir los objetivos (Cfr. Cuartas Murillo, 2008).

4 Entre otros estudios los de Gold (1990) y Morrison (1997), y las publicaciones del OPCS Longitudinal Study (Moser, 1986) o del Scottish Heart Health Study (Woodward, 1992).

5 La misma información vuelve a publicarse en $A B C$, 20-4-1988 y 21-4-1988.

6 En el estudio Framinghan se incluyeron mujeres desde la primera cohorte de 1948, puede verse una revisión de estos trabajos en María José Ruiz Somavilla, (en prensa). 


\section{Los autores}

María José Ruiz Somavilla es Profesora Titular de Historia de la Ciencia. Sus estudios sobre generización de la ciencia y de la actividad médico-sanitaria y sobre higiene en el mundo moderno han dado lugar a publicaciones como autora y colaboradora.

Gabriel Narbona Carrión es Licenciado en Medicina y Cirugía. Especialista en Medicina Familiar y Comunitaria. Ha sido director, coordinador y ponente de cursos sobre pacientes críticos y medicina de urgencias y emergencias.

María del Carmen Agüera Urbano es Licenciada en Medicina y Cirugía. Especialista en Medicina Familiar y Comunitaria. Diplomada en Salud Pública y Género. Coautora del "Protocolo andaluz de actuación sanitaria desde el ámbito de las Urgencias ante la violencia de género". 